ARTICLE

https://doi.org/10.1038/s41467-020-16752-1

\title{
Non-thermal resistive switching in Mott insulator nanowires
}

Yoav Kalcheim ${ }^{1 凶}$, Alberto Camjayi ${ }^{2}$, Javier del Valle ${ }^{1}$, Pavel Salev ${ }^{1}$, Marcelo Rozenberg ${ }^{3}$ \& Ivan K. Schuller ${ }^{1}$

Resistive switching can be achieved in a Mott insulator by applying current/voltage, which triggers an insulator-metal transition (IMT). This phenomenon is key for understanding IMT physics and developing novel memory elements and brain-inspired technology. Despite this, the roles of electric field and Joule heating in the switching process remain controversial. Using nanowires of two archetypal Mott insulators $-\mathrm{VO}_{2}$ and $\mathrm{V}_{2} \mathrm{O}_{3}$ we unequivocally show that a purely non-thermal electrical IMT can occur in both materials. The mechanism behind this effect is identified as field-assisted carrier generation leading to a doping driven IMT. This effect can be controlled by similar means in both $\mathrm{VO}_{2}$ and $\mathrm{V}_{2} \mathrm{O}_{3}$, suggesting that the proposed mechanism is generally applicable to Mott insulators. The energy consumption associated with the non-thermal IMT is extremely low, rivaling that of state-of-the-art electronics and biological neurons. These findings pave the way towards highly energyefficient applications of Mott insulators.

\footnotetext{
${ }^{1}$ Department of Physics and Center for Advanced Nanoscience, University of California-San Diego, La Jolla, CA 92093, USA. ${ }^{2}$ Departamento de Física, FCEyN, UBA and IFIBA, Conicet, Pabellón 1, Ciudad Universitaria, Buenos Aires 1428 CABA, Argentina. ${ }^{3}$ Université Paris-Saclay, CNRS, Laboratoire de Physique des Solides, 91405 Orsay, France. ${ }^{凶}$ email: ykalchheim@physics.ucsd.edu
} 
T he insulator-metal transition (IMT) in strongly correlated materials is a phase transition characterized by drastic changes in electrical properties, which may be accompanied by structural and magnetic transitions. The richness of this phenomenon has made it one of the most studied topics in condensed matter physics. The properties of IMT materials and their sensitivity to external stimuli make them promising for applications, such as memory, selectors for ReRAM, optical switches, and emulating brain functionalities ${ }^{1-17}$. Another advantage of these systems is that the IMT can be triggered by various perturbations, such as optical pumping or changes in temperature, pressure, strain, and chemical doping. The most practical way of inducing the IMT in electronic devices is by applying electrical current or voltage ${ }^{18,19}$. Such devices can be very fast ${ }^{6,20}$ and highly scalable ${ }^{17}$.

The mechanism behind the electrically triggered IMT is currently under heavy debate in the scientific community. In materials where the IMT takes place as a function of temperature, such as $\mathrm{VO}_{2}$ and $\mathrm{V}_{2} \mathrm{O}_{3}$, Joule heating due to current flow is an obvious candidate for triggering the transition. It has been argued, however, that the electric field applied in this process may induce the IMT non-thermally, without heating the material to its IMT temperature $\left(T_{\mathrm{IMT}}\right)^{21-27}$. Despite many studies, the switching mechanism is not yet understood. Even for the same materials, in some cases Joule heating is invoked, while in others non-thermal effects of the electric field may play an important role ${ }^{20-36}$. In a few studies, field-assisted carrier generation has been invoked as a possible driving mechanism for this non-thermal IMT, but no clear evidence linking the two phenomena was shown.

If non-thermal switching is indeed possible, it may have significant advantages for energy consumption, mechanical stability, and time scales as compared to the Joule heating scenario. The greatest challenge in distinguishing between the effect of Joule heating and electric field is in determining the spatial distribution of current and temperature in a device. Because of the low resistivity of metallic domains compared to insulating ones, current flow can become highly non-uniform, resulting in inhomogeneous Joule heating and electric field profiles. For example, in quasi-2D $\mathrm{VO}_{x}$ thin films, the current flow is filamentary which can dramatically alter the local temperature distribution ${ }^{19,37}$. This hinders the determination of the mechanism behind the electrically triggered IMT, since it is unclear whether the transition occurs because the IMT temperature is locally attained under an applied field.

In this work, we study the electrically triggered IMT in quasi1D nanowires of two archetypal Mott insulators exhibiting an IMT: $\mathrm{VO}_{2}$ and $\mathrm{V}_{2} \mathrm{O}_{3}$. Through accurate temperature calibration, we find that in both materials thermal and purely non-thermal IMTs are possible, thereby settling apparent contradictions regarding the origin of the field-induced IMT. We clearly identify the crucial role of defects in determining the switching mechanism and demonstrate a means of controlling it. Our findings show that defects enhance the efficiency of field-assisted carrier generation, thereby enabling a non-thermal doping driven IMT. The similarity between the switching properties in $\mathrm{VO}_{2}$ and $\mathrm{V}_{2} \mathrm{O}_{3}$, despite their different ground states, suggests that this mechanism is applicable to many other Mott insulators. By avoiding heat dissipation and its associated dynamics, the energy consumption and operation timescales of IMT-based devices may be greatly reduced.

\section{Results}

We employed advanced fabrication techniques to prepare highquality nanowires of $\mathrm{VO}_{2}$ and $\mathrm{V}_{2} \mathrm{O}_{3}$ (see scanning electron micrograph in Fig. 1a and "Methods" section). Our samples show extremely sharp 4-6 orders of magnitude resistance change across the IMT (Supplementary Fig. 1a, b). The nanowire widths $(\sim 100 \mathrm{~nm})$ are comparable to the size of insulator/metal domains, as found in a previous study ${ }^{38}$, and further supported by resistor network simulations (Supplementary Note 1 and Fig. $1 \mathrm{c}-\mathrm{e})^{1}$. The advantage of using such quasi-1D structures is that spatial confinement suppresses filament formation during the electrically triggered IMT. This greatly simplifies data interpretation and facilitates a clear distinction between thermal and electric field effects. Another advantage, as compared to the nano-gap geometry, is that nanowires are robust to power surges and thermal runaway upon nucleation of metallic domains because the remaining non-switched insulating domains act as current limiting series resistors (see Supplementary Note 3 and footnote 1). Considering homogeneous resistivity and current distributions in the nanowire, the effect of Joule heating on the nanowire temperature $\left(T_{\text {wire }}\right)$ can be quantitatively determined from a simple heat equation:

$$
C \frac{\mathrm{d} T_{\text {wire }}}{\mathrm{d} t}=I V+\kappa\left(T_{0}-T_{\text {wire }}\right)
$$

where $I$ and $V$ are the current and voltage applied to the nanowire, $C$ is its heat capacitance, $T_{0}$ is the (measured) substrate temperature and $\kappa$ is the thermal coupling constant between the substrate and the nanowire. Equation (1) is strictly valid when resistivity, power, and temperature variations along the nanowire can be neglected. This is the case in the single-phase homogeneous metallic and insulating states, above and below the IMT hysteresis. As will be shown subsequently, empirically, this equation still provides a very good approximation even when the two states coexist. In steady state $\frac{\mathrm{d} T_{\text {wire }}}{\mathrm{d} t}=0$ and thus

$$
T_{\text {wire }}=P / \kappa+T_{0}
$$

where the power is $P=I V$. To measure $\kappa$ we performed a series of voltage vs. current measurements, $V(I)$, at different $T_{0}$ corresponding to the metallic state. From this we derived resistance vs. power curves $-R(P)$, which show a trend of increasing $R$ with $P$ (see Fig. 1b). A similar trend is observed in resistance vs. substrate temperature curves which were measured at thermal equilibrium $\left[R_{\text {eq }}(T)\right]$ by applying minimal current/power to the sample and changing the stage temperature. Using Eq. (2) to relate $P$ and $T_{\text {wire }}$, we successfully collapsed all the $R(P)$ curves onto the $R_{\mathrm{eq}}(T)$, as shown in Fig. 1c. We note that all curves were collapsed using a single fitting parameter $\kappa$. For the nanowires discussed in this work, $\kappa$ ranged between 21 and $24 \mu \mathrm{W} \mathrm{K} \mathrm{K}^{-1}$ for the $\mathrm{V}_{2} \mathrm{O}_{3}$ devices and $\sim 45 \mu \mathrm{W} \mathrm{K}{ }^{-1}$ for the $\mathrm{VO}_{2}$ device (see Supplementary Note 2 for comparison of $\kappa$ values obtained in previous work and footnote 1). Knowing these thermal coupling constants allows us to accurately quantify Joule heating and enables a reliable estimate of $T_{\text {wire }}$ from the measured parameters $T_{0}$ and $P$.

To explore the thermal and electric field effects on the electrically induced IMT, current-controlled $V(I)$ measurements were performed in the low-temperature insulating state of the nanowires. For each $V(I)$, the sample was initially cooled below the IMT hysteresis regime and then heated to $T_{0}$ to allow comparison with the heating branch of the $R_{\mathrm{eq}}(T)$ measurement. The $R(P)$ curves for the $\mathrm{VO}_{2}$ nanowire in the low-temperature insulating regime are shown in Fig. 2a. A series of abrupt resistance jumps is observed in the $R(P)$ curves for $T_{0}>337 \mathrm{~K}$ (light green curve) and are due to portions of the nanowire undergoing the IMT due to the applied current. These switching events occur with decreasing power as $T_{0}$ increases. Due to the nanowire geometry, filamentary conduction does not occur so that domains evolve similarly to a temperature-driven IMT (see Supplementary Note 1). This enables a comparison between the $R(P)$ and $R\left(T_{\text {eq }}\right)$. Using only the $\kappa$ value measured above the IMT, we used Eq. (2) to 
a

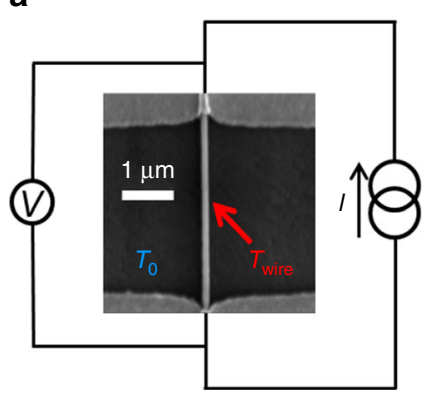

b

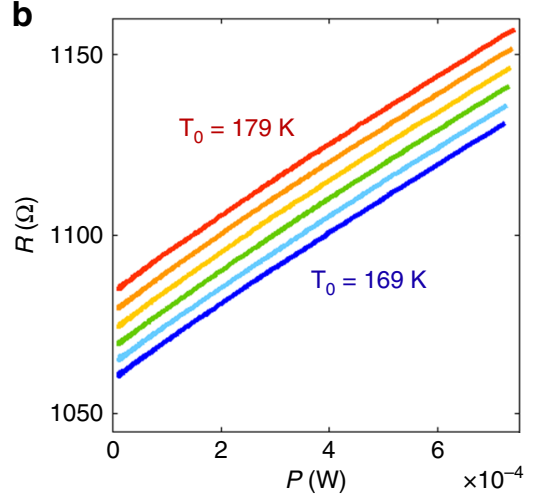

C

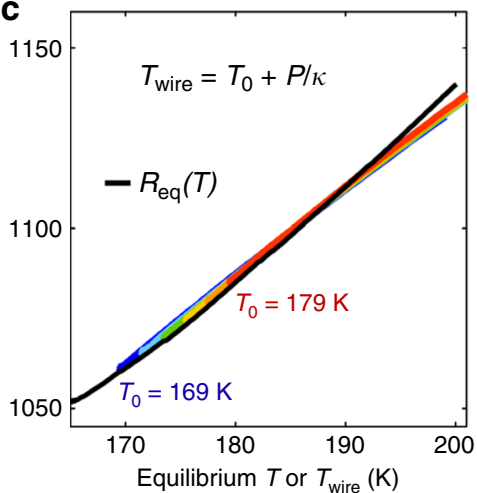

Fig. 1 Determination of temperature change due to applied power in VOx nanowires. a SEM image of a $\mathrm{V}_{2} \mathrm{O}_{3}$ nanowire contacted by two Ti/Au pads (top and bottom) and schematic of the measurement setup. $T_{0}$ and $T_{\text {wire }}$ are the temperatures of the substrate and the nanowire, respectively, which differ due to the applied power. $\mathbf{b}$ Resistance vs. power curves measured above the IMT temperature in the fully metallic state of a $V_{2} \mathrm{O}_{3}$ nanowire at $T_{0}$ between 169 and $179 \mathrm{~K}$ (blue to red). c Resistance vs. $T_{\text {wire }}$ (colored lines) derived from $\mathbf{b}$ using Eq. (2) with a single fitting parameter $\kappa=24 \mu \mathrm{W} \mathrm{K}-1$ for all curves (colored). The black curve shows the resistance vs. stage temperature at equilibrium, $R_{\mathrm{eq}}(T)$. The horizontal $T$ axis refers both to the stage temperature during the equilibrium (low power) measurement and $T_{\text {wire. }}$.

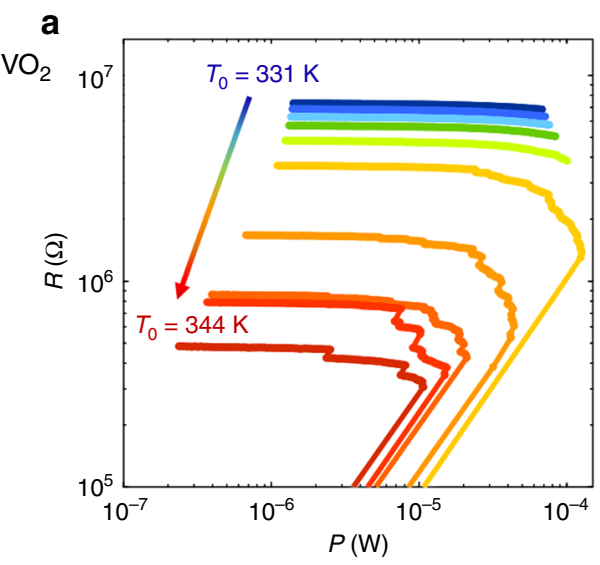

C

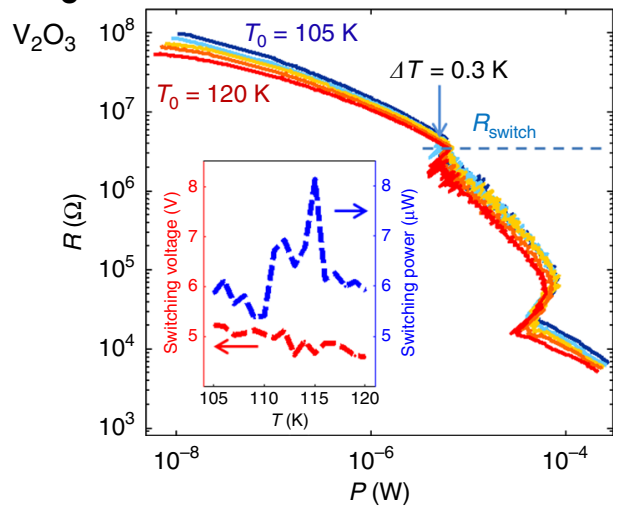

b

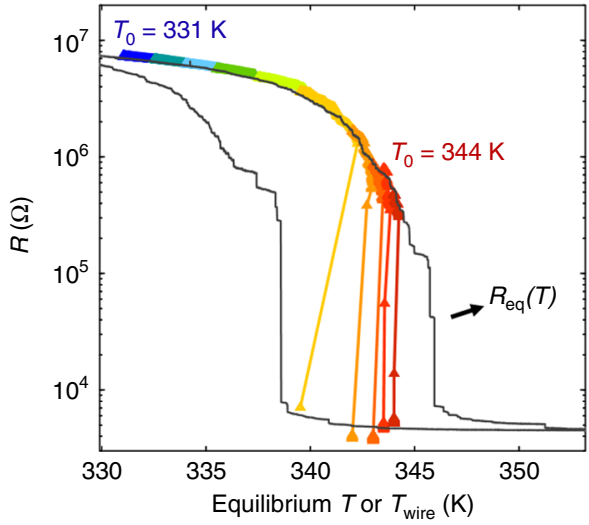

d

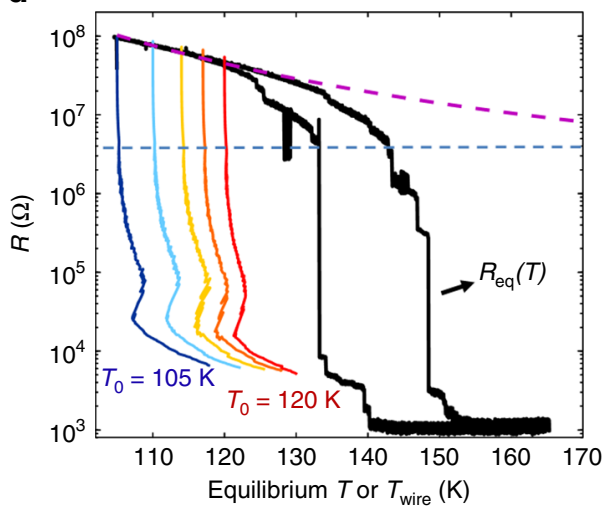

Fig. 2 Thermal vs. non-thermal IMT switching. a and $\mathbf{c} R(P)$ curves acquired at different substrate temperatures, $T_{0}$, corresponding to the insulating state of $\mathrm{VO}_{2}$ and $\mathrm{V}_{2} \mathrm{O}_{3}$ nanowires, respectively. The IMT switching is manifested as abrupt resistance jumps. $\mathbf{b}$ and $\mathbf{d} R\left(T_{\text {wire }}\right)$ curves derived from the corresponding $R(P)$ curves and superimposed on the $R_{\mathrm{eq}}(T)$ (black). For the $\mathrm{VO}_{2}$ nanowire in $\mathbf{b}$, the excellent correspondence between $R\left(T_{\text {wire }}\right)$ and $R_{\mathrm{eq}}(T)$ signifies that the IMT is triggered thermally. For the $\mathrm{V}_{2} \mathrm{O}_{3}$ nanowire in $\mathbf{d}$, no such correspondence between $R\left(T_{\text {wire }}\right)$ and $R_{\mathrm{eq}}(T)$ is observed, showing the inadequacy of a Joule-heating driven mechanism. The IMT switching in $\mathbf{c}$ begins with an increase of temperature $\Delta T=0.3 \mathrm{~K}$ in $T_{\text {wire }}$ regardless of $T_{0}$ (light blue arrow), indicating a negligible role of Joule heating. The switching resistance $R_{\text {switch }}$ (dashed blue line) is lower than the fully insulating state resistance at any temperature. This is shown in $\mathbf{d}$ by the extrapolation of $R_{\mathrm{eq}}(T)$ according to thermally activated resistance behavior with an activation energy of $60 \mathrm{meV}$ (purple dashed line). The inset in c shows the switching voltage and power as functions of $T_{0}$. 
transform the $R(P)$ curves measured at different $T_{0}$ in the insulating state to $R\left(T_{\text {wire }}\right)$. Figure $2 \mathrm{~b}$ shows that the $R\left(T_{\text {wire }}\right)$ curves collapse onto the $R\left(\mathrm{~T}_{\mathrm{eq}}\right)$, similar to the collapse observed in the metallic state (see Fig. 1a, c). The collapse, extending well into the IMT, shows that electrical switching in this $\mathrm{VO}_{2}$ nanowire can be accounted for solely by Joule heating. These findings are consistent with most previous reports which suggest that Joule heating plays a dominant role in the electrically induced IMT in $\mathrm{VO}_{2}{ }^{21,30,39,40}$. The large jumps in the last section of some $R\left(T_{\text {wire }}\right)$ curves are attributable to thermal runaway effects due to a current surge in the nanowire (see Supplementary Note 3 and footnote 1).

We note that our temperature calibration is strictly valid only in the homogeneous states (fully metallic or insulating). However, it is found that $R\left(T_{\text {wire }}\right)$ collapses onto the $R_{\mathrm{eq}}(T)$ even when some metallic domains are present in the mostly insulating nanowire. This indicates that the temperature is approximately constant along the nanowire despite spatial variations in heat dissipation. This is due to thermal diffusion within the nanowire which tends to smear temperature variations. For higher power/temperature most of the sample is metallic resulting in a highly inhomogeneous temperature profile. To adequately describe this state local dissipation and spatial derivative terms have to be considered. However, for the purpose of identifying the mechanism behind the electrical IMT, we focus on the initial jump in the low power/ temperature regime where Eq. (1) is valid.

Considering the electrically induced IMT switching shown in Fig. 2a, b as a benchmark for a purely Joule heating driven transition, we show that the $\mathrm{V}_{2} \mathrm{O}_{3}$ sample discussed in Fig. $2 \mathrm{c}$, $\mathrm{d}$ exhibits pronounced qualitative differences. To assess the role of Joule heating, we follow the same procedure as before to calculate $R\left(T_{\text {wire }}\right)$ from the measured $R(P)$ in the metallic state and Eq. (2). The $R(P)$ and corresponding $R\left(T_{\text {wire }}\right)$ curves in the insulating state of this $\mathrm{V}_{2} \mathrm{O}_{3}$ nanowire are shown in Fig. 2c, d, respectively. The stark differences between these results and those of the $\mathrm{VO}_{2}$ sample shown in Fig. 2a, b, indicate that the thermal model is inadequate for describing the evolution of resistance with power in this $\mathrm{V}_{2} \mathrm{O}_{3}$ nanowire. In the following, we show that this is due to two phenomena which occur with negligible change in temperature: a smooth decrease in resistance and subsequent abrupt resistive switching. Initially, $R(P)$ decreases smoothly by over an order of magnitude with increasing power up to $\sim 5-8 \mu \mathrm{W}$. As can be deduced from comparison of the $R\left(T_{\text {wire }}\right)$ to $R_{\text {eq }}(T)$, the voltage-driven decrease in resistance is not due to Joule heating, since the dissipated power is too low (Fig. 2d). This smooth decrease is not attributed to the IMT either, but rather to fieldassisted carrier generation, as will be discussed subsequently. After $R$ decreases smoothly down to 3-5 $\mathrm{M} \Omega$ it drops abruptly by $20-30 \%$. It is tempting to immediately attribute this abrupt resistance drop to the IMT, but before we may safely do so, we have to rule out other possibilities. Besides an electrically driven IMT, resistive switching can be due to ionic migration, thermally induced negative differential resistance (NDR), and ovonic switching. Our recent work demonstrated oxygen migration in vanadium oxides under high applied electric field ${ }^{10}$. This process changes the material stoichiometry resulting in abrupt nonvolatile resistive switching, which can only be reversed by hightemperature annealing. In contrast, the resistive switching in our device is volatile at temperatures below the IMT hysteresis (i.e. the initial resistance recovers when the current is ramped down), thus ruling out oxygen migration as the cause for the resistance jumps. Thermally induced NDR can also result in abrupt resistive switching before the onset of IMT in materials such as $\mathrm{NbO}_{2}{ }^{41,42}$. Such an NDR is due to a steep resistance-temperature dependence and appears with very significant Joule heating $(\Delta T \sim$ $100 \mathrm{~K})$. In our devices, we deduce that the temperature increases by only $\sim 0.3 \mathrm{~K}$ from the power-to-temperature calibration (see also details on Joule heating dynamics in our samples in Supplementary Note 3) (see footnote 1), which is inconsistent with a thermally induced NDR. Finally, non-thermal ovonic switching can also produce an abrupt resistance jump ${ }^{43}$. This type of threshold switching is due to carrier generation and saturation of charge traps under the application of a strong electric current. When the current is ramped down below a certain holding current, the device reverts back to the high resistance state. However, when switching within the hysteretic temperature range of our devices, the initial high resistance state is not recovered, even at zero applied current. This shows that portions of the nanowire have undergone the IMT and remain in the metallic state because it is thermodynamically stable at these temperatures. After ruling out other possibilities for the observed threshold resistive switching, we conclude that the resistance jumps in Fig. $2 \mathrm{c}$ are indeed due to an electrically driven IMT.

As noted above, the power just before the first switching event $(\sim 7 \mu \mathrm{W})$ heats the nanowire by only $\sim 0.3 \mathrm{~K}$, despite a $15 \mathrm{~K}$ range in $T_{0}$. Moreover, the switching power is nearly independent of $T_{0}$, and varies in the range $5-8 \mu \mathrm{W}$ (see inset in Fig. 2c). These findings are inconsistent with Joule heating-driven switching, for which the switching power would decrease with increasing $T_{0}$ by $\sim 21 \mu \mathrm{W} \mathrm{K} \mathrm{K}^{-1}$ (as deduced from power-to-temperature calibration). We therefore conclude that the switching observed in this sample is due to a non-thermal, field-driven IMT.

Interestingly, the resistive switching properties of different $\mathrm{V}_{2} \mathrm{O}_{3}$ nanowires showed some variability, most likely induced during the fabrication process. A systematic comparison of these properties allowed us to gain insight into the underlying mechanism of the electric-field-driven IMT. Figure 3a shows $R_{\text {eq }}(T)$ curves of four $\mathrm{V}_{2} \mathrm{O}_{3}$ nanowires. The IMT in all four nanowires occurs over virtually the same temperature range. We find, however, that for any fixed temperature below the IMT hysteresis, the equilibrium insulating-state resistance varies between the nanowires. For instance, the resistance at $120 \mathrm{~K}$ $\left(R_{120 \mathrm{~K}}\right)$ in the heating branch of the $R_{\mathrm{eq}}(T)$ curves varies from 46 to $1100 \mathrm{M} \Omega$. Since the nanowire geometries are nearly identical, these resistance variations are most likely due to properties of defects. Defects may create in-gap trap states that release carriers by thermal activation. Thus, the defect concentration and activation energy determine the amount of carriers which can be excited into the conduction band, thereby strongly influencing the insulating state resistance ${ }^{44}$. Indeed, recent studies have shown that defects due to deviations from perfect stoichiometry decrease the insulating state resistivity in $\mathrm{VO}_{2}$ and $\mathrm{V}_{2} \mathrm{O}_{3}{ }^{45,46}$. In our $\mathrm{V}_{2} \mathrm{O}_{3}$ samples we also find that $R_{120 \mathrm{~K}}$ increases monotonously with the activation energy (see Supplementary Note 4 and footnote 1 ). We thus use $R_{120 \mathrm{~K}}$ as an inverse proxy for the amount of carriers which can be generated in the nanowires.

Based on this analysis, we now show that defects play a crucial role in determining the non-equilibrium properties of $\mathrm{V}_{2} \mathrm{O}_{3}$ and, consequently, affect the mechanism by which the IMT takes place. To elucidate this, typical $R(V)$ curves for the samples of Fig. 3a are shown in Fig. 3b. For comparison, three $R(V)$ curves were measured at different $T_{0}$ so that they have the same initial resistance (blue, green, and red). This eliminates the influence of initial resistance and enables a fair comparison between the samples. Two important effects are observed for samples with lower $R_{120 \mathrm{~K}}$ : (1) the initial slope of the smooth part of $\log [R(V)]$ curve becomes steeper and (2) the switching voltage $\left(V_{\text {switch }}\right)$ decreases. Unexpectedly, we find that $V_{\text {switch }}$ is lower in measurements performed at lower temperatures, as shown in Fig. $3 \mathrm{~b}$. This points to the dominant role of defects in determining switching properties rather than the proximity of $T_{0}$ to $T_{\text {IMT }}$. For instance, the sample with $R_{120 \mathrm{~K}}=46 \mathrm{M} \Omega$ switches with only $\sim 5.2 \mathrm{~V}\left(1.5 \mathrm{MV} \mathrm{m}^{-1}\right)$ and $\sim 6 \mu \mathrm{W}$ at $T_{0}=105 \mathrm{~K}$, well below $T_{\text {IMT }}$. 

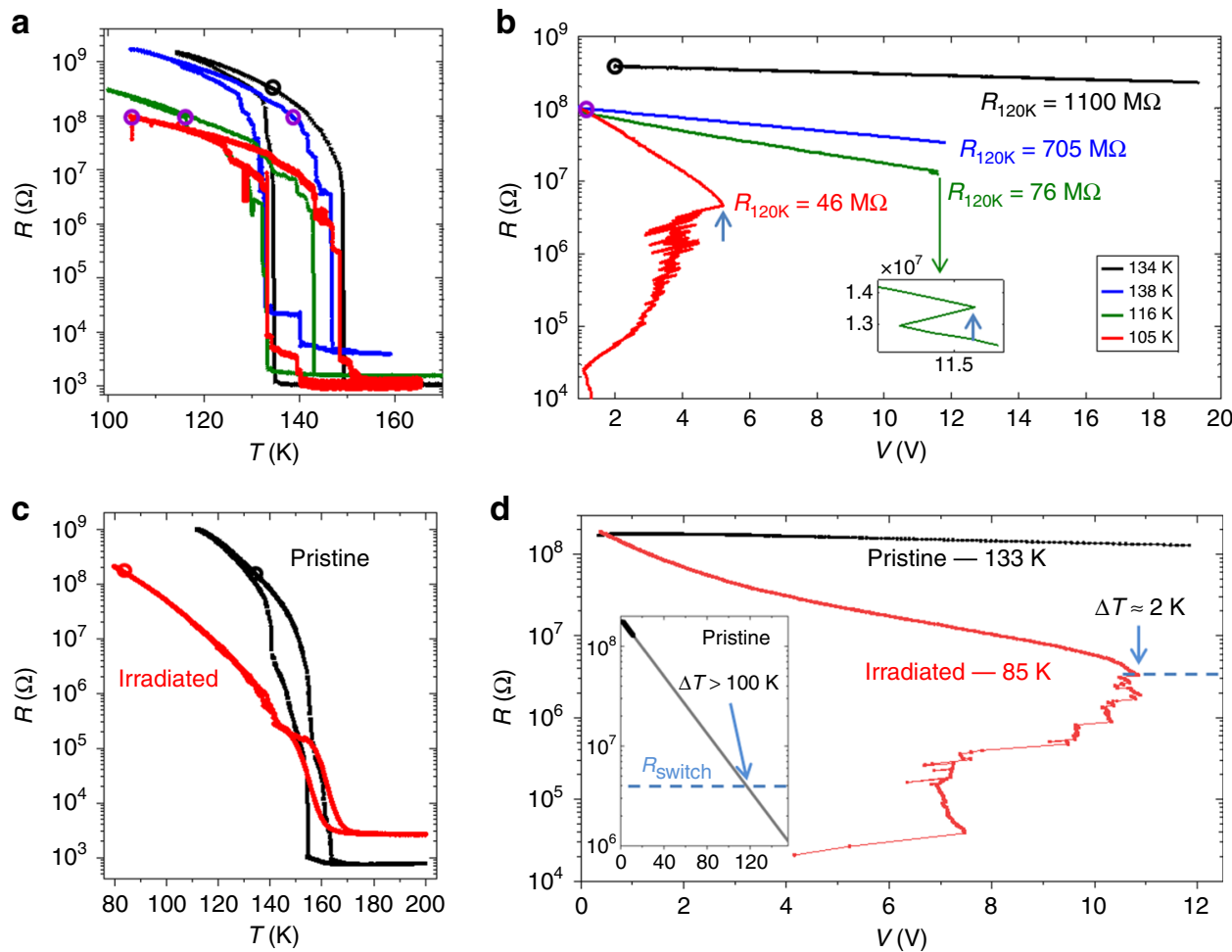

Fig. 3 Influence of defects on the switching mechanism. a Resistance vs. temperature curves of four $\mathrm{V}_{2} \mathrm{O}_{3}$ nanowires. Open circles denote $T_{0}$ for which the $R(V)$ curves shown in $\mathbf{b}$ were acquired. $\mathbf{b} R(V)$ for each of the nanowires in $\mathbf{a}$ with the corresponding color coding. $T_{0}$ is indicated in the legend. Samples with lower insulating state resistance, $R_{120 \mathrm{~K}}$, exhibit steeper slopes of $\log (R)$ vs. voltage, indicating enhanced carrier generation. For two samples, abrupt resistance jumps associated with the IMT are observed (light blue arrows). $\mathbf{c} R_{\text {eq }}(T)$ before and after focused ion beam irradiation with a dose of $6.2 \times 10^{14}$ $\mathrm{Ga}$ ions $\mathrm{cm}^{-2}$. Open circles denote $T_{0}$ for which the $R(V)$ curves shown in $\mathbf{d}$ were acquired. $\mathbf{d} R(V)$ measured in the pristine and irradiated states. Similar to b, the slope of $\log (R)$ vs. voltage is considerably steeper in the irradiated state. After the smooth decrease, the resistance reaches $2 \%$ of the initial value, $R_{\text {switch }}=4 \mathrm{M} \Omega$, followed by non-thermal switching with a temperature increase $\Delta T \sim 2 \mathrm{~K}$. For the pristine state, assuming the same $R_{\text {switch }}=4 \mathrm{M} \Omega$, an extrapolation of $R(V)$ predicts that switching occurs with over $110 \mathrm{~V}$ corresponding to a temperature increase of more than $100 \mathrm{~K}$ (inset to d).

In contrast, the sample with $R_{120 \mathrm{~K}}=705 \mathrm{M} \Omega$ does not switch up to $12 \mathrm{~V}\left(3.4 \mathrm{MV}^{-1}\right)$ with similar applied power at $T_{0}=138 \mathrm{~K}$, despite being inside the phase coexistence regime. The sample with the highest $R_{120 \mathrm{~K}}=1100 \mathrm{M} \Omega$ does not switch even with application of $20 \mathrm{~V}$. The non-equilibrium properties are thus observed to be strongly dependent on the insulating state resistance, which is strongly affected by defects in the nanowire.

To examine this further, we used focused $\mathrm{Ga}$ ion beam irradiation to systematically and controllably create defects in our samples. Only the nanowire area was exposed to the ion beam with minimal exposure to the contacts, so that the resulting changes in transport properties can only be attributed to the nanowire itself. Figure $3 \mathrm{c}$ shows the $R_{\mathrm{eq}}(T)$ curves of a $\mathrm{V}_{2} \mathrm{O}_{3}$ nanowire before and after ion irradiation. Similar to the samples shown in Fig. 3a, defects reduce the insulating state resistance but do not considerably affect the temperature range over which the IMT takes place. As in the non-irradiated samples, the $R(V)$ characteristics show dramatic differences due to the varying defect density. Figure $3 \mathrm{~d}$ shows two $R(V)$ measurements acquired at $T_{0}=133$ and $85 \mathrm{~K}$ in the pristine and irradiated states, respectively, so that the initial resistance is the same in both cases. For the pristine state, the resistance smoothly decreases to $73 \%$ of the initial value for the maximum voltage, while the resistance of the irradiated sample smoothly decreases by almost two order of magnitude to $\sim 2 \%$ of its initial value, followed by an abrupt resistance jump at $R_{\text {switch }}=4 \mathrm{M} \Omega$ (light blue arrow). Just before switching, Joule heating in the nanowire corresponds to a temperature increase of $<2 \mathrm{~K}\left(T_{\text {wire }}<87 \mathrm{~K}\right)$. However, the first signatures of the IMT in $R_{\text {eq }}(T)$ occur at $\sim 120 \mathrm{~K}$, which would require an increase in $T_{\text {wire }}$ of $\sim 35 \mathrm{~K}$. Therefore, this switching cannot be explained by thermal effects. Qualitatively similar results were obtained also on irradiated $\mathrm{VO}_{2}$ nanowires (see Supplementary Note 5 and footnote 1), thus corroborating the important role of defects in facilitating the electrically driven nonthermal IMT in Mott insulators.

\section{Discussion}

Zener-tunneling is a well-studied mechanism for field-induced doping and destabilization of Mott insulators ${ }^{47-51}$. Within this mechanism, the electric field is high enough $\left(70-400 \mathrm{MV} \mathrm{m}^{-1}\right)$ to enable tunneling of carriers across the Mott gap, which drives a doping driven transition. In our work, the switching fields are much smaller $\left(<3 \mathrm{MV} \mathrm{m}^{-1}\right)$ so that Zener-tunneling can be ruled out. However, a similar end result can be achieved by fieldassisted activation of carriers from trap-states. Instead of tunneling through the gap, charge trapped in defect in-gap states can be activated and provide enough carriers to dope the Mott insulator and induce the IMT. Previous theoretical works have shown that impurities can dramatically reduce the switching field with respect to the one expected from Zener tunneling 52,53 . In the following, we discuss how defects increase field-assisted carrier emission in our samples, which in turn enables a non-thermal doping-driven IMT with low field.

First, we discuss the origin of the initial smooth decrease in resistance in response to the applied voltage as shown in Fig. 3b, d. This decrease cannot be attributed to the IMT since switching even in a single domain would produce a resistance jump due to 

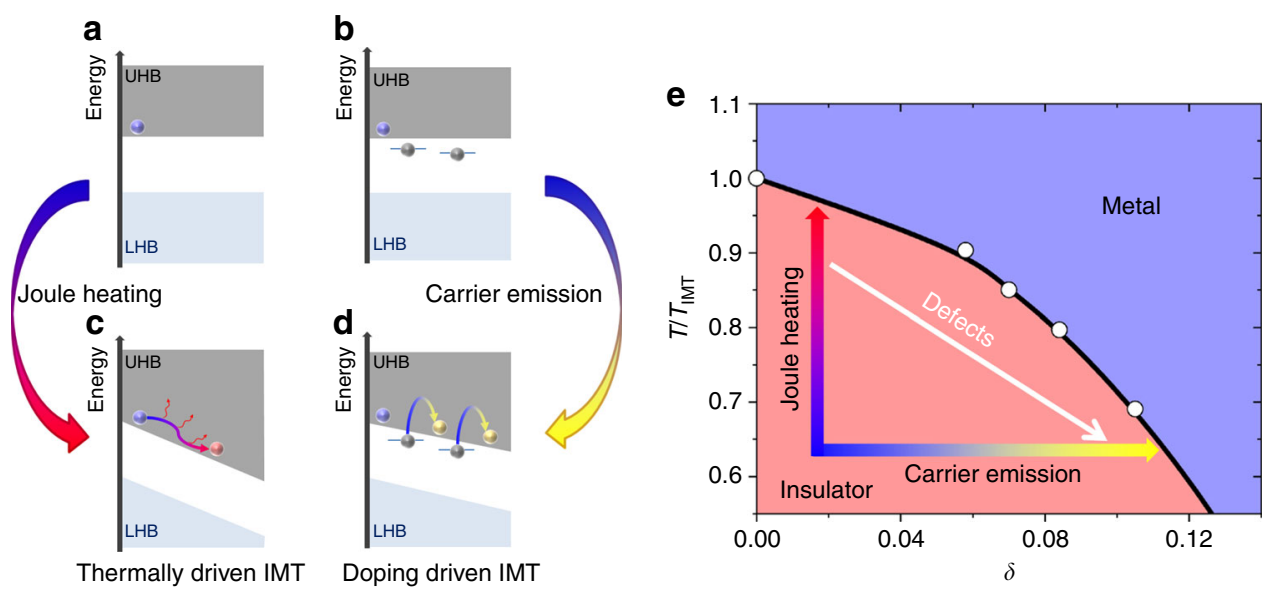

Fig. 4 Two routes for resistive switching in a Mott insulator. a-d Schematic representation of Joule heating and field-driven IMT switching in a Mott insulator. In a system with no defects $\mathbf{a}$, only a small number of thermally activated carriers are present in the upper Hubbard band (UHB). Application of a strong electric field, depicted as strong band tilt $\mathbf{c}$, accelerates these carriers causing Joule heating due to scattering. In a system with defects $\mathbf{b}$, a moderate electric field promotes carriers from in-gap states into the UHB $\mathbf{d}$ resulting in collapse of the Mott insulator state via a doping-driven IMT. e Phase diagram of the single band Hubbard model based on DMFT calculations. The colored arrows schematically denote the two possible routes towards the metallic phase when applying electric field as discussed in a-d. Defects increase the effectiveness of carrier emission and facilitate a doping driven nonthermal IMT.

the low dimensionality of the nanowire, which is not observed. Furthermore, hysteretic behavior is not observed in the $R(V)$ curves within the initial voltage-driven smooth decrease of $R$, even at temperatures within the phase coexistence regime. Similar voltage-driven decrease in resistance has been previously observed in Mott insulators ${ }^{20,54}$ and attributed to field-assisted carrier generation. One mechanism for this is the Poole-Frenkel effect $^{55}$, in which the electric field reduces the energy barrier for excitation of trapped carriers from in-gap states into the upper Hubbard band (Fig. 4b, d). We note that only carrier excitation into the upper Hubbard band is shown in Fig. 4 but carriers may also be excited into other unoccupied bands. For example, in lowtemperature insulating $\mathrm{V}_{2} \mathrm{O}_{3}$, the $\mathrm{a}_{1 \mathrm{~g}}$ band, which belongs to the lower Hubbard bands, is above the Fermi level and, consequently, is unoccupied ${ }^{56}$. As a function of the electric field $(E)$, the energy barrier may decrease linearly with $E$ or $E^{1 / 2} 57$, producing a large increase in carrier density, consistent with the observed decrease of resistance with voltage (see also Supplementary Note 6 for details and fitting to the Poole-Frenkel conduction enhancement) (see footnote 1). The slope of these curves steepens with decreasing $R_{120 \mathrm{~K}}$, indicating that defects determine the number of carriers which are excited for the same applied field ${ }^{58}$. As demonstrated in Supplementary Fig. 6 (see footnote 1), the resistance just before switching $\left(R_{\text {switch }}\right)$ is nearly independent of $T$ and lower than the equilibrium resistance of the fully insulating phase, even if it is extrapolated to temperatures higher than that of the IMT (Fig. 2d). Therefore, at $R_{\text {switch }}$, the effect of field is to increase the carrier concentration beyond that of the insulating state in equilibrium. This excess carrier concentration leads to destabilization of the insulating state, resulting in emergence of the metallic state. Since field-assisted carrier concentration increases with temperature, the switching voltage $\left(V_{\text {switch }}\right)$ decreases with increasing $T_{0}$ (inset to Fig. 2c). We note that other field-enhanced carrier emission processes may also lead to similar results. For instance, switching in the Ga-ion-irradiated $\mathrm{VO}_{2}$ nanowire is preceded by a conductivity enhancement according to $\ln (\sigma) \propto E^{2}$, indicative of phonon-assisted tunneling ${ }^{59}$ (see Supplementary Note 6 and footnote 1).

In samples where the electric-field-assisted carrier generation is inefficient, the doping driven transition cannot be attained even for the highest applied voltages and the only possible route to induce the IMT switching is through Joule heating past $T_{\text {IMT }}$ (Fig. $4 \mathrm{a}, \mathrm{c}$ ). This is most notable for the non-irradiated $\mathrm{VO}_{2}$ nanowires which transition thermally (Fig. 2a, Supplementary Fig. 4 and footnote 1) and non-irradiated $\mathrm{V}_{2} \mathrm{O}_{3}$ nanowires with the highest insulating state resistances (black curves in Fig. $3 \mathrm{~b}$ and d). For these $\mathrm{V}_{2} \mathrm{O}_{3}$ samples, even up to the highest applied voltages, the resistance decreases very little compared to samples with low $R_{120 \mathrm{~K}}$. Therefore, the carrier density does not reach the critical value required to induce switching in a non-destructive voltage range. We therefore assess $V_{\text {switch }}$ in these samples by extrapolation. For instance, the inset to Fig. 3d shows a linear extrapolation of the $\log [R(V)]$ for the pristine nanowire to a resistance of $4 \mathrm{M} \Omega$-the $R_{\text {switch }}$ value observed in the same sample after irradiation. Assuming that the linear dependence holds for higher voltages, switching would occur with over $110 \mathrm{~V}$. This translates to Joule heating by over $100 \mathrm{~K}$, which would bring the sample well above $T_{\text {IMT }}$. This indicates that thermal effects would play a major role in the electrical triggering of the IMT in this sample. We therefore conclude that there is a crossover in the switching mechanism from carrier generation to Joule heating in both $\mathrm{VO}_{2}$ and $\mathrm{V}_{2} \mathrm{O}_{3}$.

We note that non-thermal switching in $\mathrm{VO}_{2}$ requires higher irradiation doses compared to $\mathrm{V}_{2} \mathrm{O}_{3}$ (see Supplementary Note 5 and footnote 1). Even with these high doses, electric-field-assisted carrier generation was not as effective as in $\mathrm{V}_{2} \mathrm{O}_{3}$, leading to higher $V_{\text {switch }}$ values in $\mathrm{VO}_{2}$. This points to the role of the activation energy which is considerably higher in $\mathrm{VO}_{2}(265 \mathrm{meV})$ compared to the $\mathrm{V}_{2} \mathrm{O}_{3}$ samples which exhibit non-thermal switching $(60 \mathrm{meV}) . V_{\text {switch }}$ also increases with increasing activation energies for the various $\mathrm{V}_{2} \mathrm{O}_{3}$ samples shown in Fig. 3 (see Supplementary Note 4 and footnote 1). We thus conclude that with higher defect densities and lower activation energy, more carriers are generated with less applied field and power, which enables non-thermal switching. In the clean limit, carrier generation is inefficient and Joule heating thermally triggers the IMT before the critical carrier concentration is attained (Fig. 4).

The strong dependence on specific sample properties can explain apparent discrepancies regarding the nature of the IMT found in previous reports on the same materials ${ }^{20,21,25,40,60}$. These reports may be reconciled by considering differences in sample properties such as resistivity, permittivity, defect density, 
and activation energy (see also Supplementary Notes 2, 6 and footnote 1). These properties are crucial for determining whether a Mott insulator will undergo thermal or non-thermal electrical switching. We note that the quasi-1D nature of our samples helps to identify the mechanism behind the electrically driven IMT by eliminating filament formation (see also discussion in Supplementary Note 3 and footnote 1). However, the sample geometry does not determine the switching mechanism. Geometry might affect the overall switching dynamics and total energy consumption but not its microscopic origin. In fact, several studies have concluded based on simulations and switching time scales that in 2D/3D devices the transition temperature is not attained during switching $21,25,27$.

To gain deeper insight into the physics of the electrically induced IMT, we performed dynamical mean field theory $(D M F T)^{61}$ calculations for a single band Hubbard model. This model is considered the simplest framework for a qualitative description of the IMT in a strongly interacting electron system (see Supplementary Note 6 and footnote 1). Motivated by our previous discussion, we assume that the main effect of the electric field is to promote charge carriers in the system, which is modeled by shifting the electric (chemical) potential to adjust the doping level. Starting from the insulator state, the resistivity $\rho(T)$ was calculated using the Kubo formula for various temperatures and doping levels to determine the regimes of insulating $(\mathrm{d} \rho / \mathrm{d} T<0)$ and metallic $(\mathrm{d} \rho / \mathrm{d} T>0)$ behavior. The goal is to qualitatively examine the resistivity across the thermal and doping-driven IMT and compare these results with our experimental findings. The calculations show that the IMT can be attained either by increasing temperature or by doping (see phase diagram in Fig. 4e). This is qualitatively consistent with the phase diagram of many Mott insulators including the cuprates ${ }^{62}, \mathrm{VO}_{2}{ }^{63}$, and $\mathrm{V}_{2} \mathrm{O}_{3}{ }^{64}$, even though these materials have a more complicated band structure than that of our model. Despite the simplicity of the model, several important characteristics of the experimental switching are reproduced by our calculations. We observe that $\rho$ decreases considerably as the sample is doped by a few percent until the system switches from insulating to metallic behavior. Moreover, it is found that $\rho$ at the switching point is lower than that of the insulating state in equilibrium and has a weak dependence on temperature, as we observe experimentally (see Supplementary Note 6). The ability of such a general and schematic model to capture important aspects of our experimental findings suggests that the proposed mechanism for the electric field-driven IMT may apply to many Mott insulators beyond $\mathrm{VO}_{2}$ and $\mathrm{V}_{2} \mathrm{O}_{3}$.

The ability to induce non-thermal switching without heating the device by more than a fraction of a Kelvin implies that this process can be highly energy efficient. Indeed, by applying nanosecond voltage pulses to the $\mathrm{V}_{2} \mathrm{O}_{3}$ nanowire discussed in Fig. $2 c$, d, an upper bound of $5 \mathrm{fJ}$ is found for the switching energy (Supplementary Note 7). This presents an improvement in energy consumption of about three orders of magnitude over the Joule heating-driven IMT and rivals resistive switching in state of the art memristors ${ }^{65}$. This has very important implications on the energetics of devices, which utilize the IMT for memory or for emulating neural functionalities. Moreover, the time scales associated with the non-thermal IMT may be much shorter than for the thermal IMT. For devices based on a thermal IMT, accumulation and dissipation of heat sets a lower bound on the operation time scales ${ }^{66}$. By avoiding heating during the switching process these time scales may be greatly reduced.

To conclude, in this work we disentangle the effect of Joule heating and electric field on resistive switching in two archetypal Mott insulators $-\mathrm{VO}_{2}$ and $\mathrm{V}_{2} \mathrm{O}_{3}$. We show clear evidence for thermal and non-thermal IMTs in both materials. Non-thermal switching occurs when field-assisted carrier generation leads to critical doping levels where the Mott insulator state is no longer stable. Several features of our experimental findings are reproduced by DMFT calculations, supporting the doping-driven IMT scenario. We find that defects are crucial for the non-thermal IMT, as they provide the charge reservoir for the doping process. To enhance their effect, large defect densities may be introduced into the sample during fabrication or by focused ion beam (FIB) irradiation. This allows moderate fields to excite a large number of carriers with negligible heating. For low defect densities, carrier generation is unable to attain critical values, leaving Joule heating as the dominant switching mechanism. Our work demonstrates that, despite having two different ground states, $\mathrm{VO}_{2}$ and $\mathrm{V}_{2} \mathrm{O}_{3}$ can be modified through defect engineering to exhibit a nonthermal IMT. This suggests that using controlled defects to induce non-thermal switching may be applicable to many Mott insulators, and greatly reduce the energy consumption and operating timescales of IMT-based devices.

\section{Methods}

Sample preparation. $\mathrm{VO}_{2}$ : A $100 \mathrm{~nm}$-thick $\mathrm{VO}_{2}$ film was grown on (012)-oriented $\mathrm{Al}_{2} \mathrm{O}_{3}$ substrate by reactive $\mathrm{rf}$ magnetron sputtering. The growth was done in a 4 mTorr $\mathrm{Ar} / \mathrm{O}_{2}(92-8 \%)$ atmosphere and at a substrate temperature of $470{ }^{\circ} \mathrm{C}$. After the growth, the sample was cooled down at a rate of $12^{\circ} \mathrm{C} \mathrm{min}-1$. X-ray diffraction showed textured growth along the (100) direction of $\mathrm{VO}_{2} . \mathrm{V}_{2} \mathrm{O}_{3}: 300 \mathrm{~nm}$-thick $\mathrm{V}_{2} \mathrm{O}_{3}$ films were grown on (012)-oriented $\mathrm{Al}_{2} \mathrm{O}_{3}$ substrates by $\mathrm{rf}$ magnetron sputtering. The growth was done in a $8 \mathrm{mTorr} \mathrm{Ar}$ atmosphere and at a substrate temperature of $700{ }^{\circ} \mathrm{C}$. After growth, the samples were thermally quenched at a rate of $\sim 90^{\circ} \mathrm{C} \mathrm{min}-1$, which significantly improved the film's electrical properties ${ }^{67}$. Xray diffraction showed textured growth along the (012) direction of $\mathrm{V}_{2} \mathrm{O}_{3}$.

For both materials, nanowires of $85-100 \mathrm{~nm}$ width were fabricated by e-beam lithography and reactive ion etching. Au $(100 \mathrm{~nm}) / \mathrm{Ti}(20 \mathrm{~nm})$ electrodes were subsequently prepared by photolithography and e-beam evaporation to electrically contact 3-3.5 $\mu \mathrm{m}$ long nanowires.

Transport measurements. Transport measurements were performed in a probe station using a current source pulse generator, nanovoltmeter, and $20 \mathrm{GHz}$ oscilloscope. To protect the sample against current surges during abrupt resistive switching, for some measurements a resistor was connected in series. Fast transport measurements to determine the energy consumption during nonthermal switching were performed using a bias tee to mix nanosecond pulses with a small DC current. The DC current was used to probe the resistance of the sample before and after the pulse. Due to the large impedance mismatch, a $50 \Omega$ termination was added at the output of the pulse generator to avoid reflection.

FIB irradiation. Ion beam irradiation was performed in a FEI Scios DualBeam system using a FIB of $30 \mathrm{keV}$ Ga ions. The etch rate was calibrated by measuring the dose required to etch the vanadium oxide film all the way to the substrate. For the dose used in this work $\left(6.2 \times 10^{14}\right.$ ions $\left.\mathrm{cm}^{-2}\right)$, the etching depth is estimated at $\sim 1.4 \AA$, so that the film thickness is practically unaffected. The nanowire region was irradiated by scanning the FIB at $1.5 \mathrm{pA}$ with $50 \%$ overlap between successive locations to ensure a uniform irradiation dose across the entire nanowire.

\section{Data availability}

All relevant data is available from the corresponding author upon request.

\section{Code availability}

All code used in simulations supporting this article is available from Y.K. (ykalchheim@ucsd.edu), J.d.V. (jdelvallegranda@ucsd.edu), or M.R. (marcelorozenberg@u-psud.fr) upon request.

Received: 29 January 2020; Accepted: 20 May 2020; Published online: 12 June 2020

\section{References}

1. Bae, S. H. et al. The memristive properties of a single $\mathrm{VO} 2$ nanowire with switching controlled by self-heating. Adv. Mater. 25, 5098-5103 (2013). 
2. Pellegrino, L. et al. Multistate memory devices based on free-standing VO2/ $\mathrm{TiO} 2$ microstructures driven by joule self-heating. Adv. Mater. 24, 2929-2934 (2012).

3. Shukla, N. et al. A steep-slope transistor based on abrupt electronic phase transition. Nat. Commun. 6, 7812 (2015).

4. Ignatov, M., Ziegler, M., Hansen, M., Petraru, A. \& Kohlstedt, H. A memristive spiking neuron with firing rate coding. Front. Neurosci. 9, 376 (2015).

5. Driscoll, T., Kim, H.-T., Chae, B.-G., Di Ventra, M. \& Basov, D. N. Phasetransition driven memristive system. Appl. Phys. Lett. 95, 43503 (2009).

6. Yang, Z., Ko, C. \& Ramanathan, S. Oxide electronics utilizing ultrafast metal-insulator transitions. Annu. Rev. Mater. Res. 41, 337-367 (2011).

7. Jerominek, H., Picard, F. \& Vincent, D. Vanadium oxide films for optical switching and detection. Opt. Eng. 32, 2092-2099 (1993).

8. Butakov, N. A. et al. Switchable plasmonic-dielectric resonators with metal-insulator transitions. ACS Photonics 5, 371-377 (2018).

9. Son, M. et al. Excellent selector characteristics of nanoscale VO2 for highdensity bipolar ReRAM applications. IEEE Electron Device Lett. 32, 1579-1581 (2011).

10. del Valle, J. et al. Electrically induced multiple metal-insulator transitions in oxide nanodevices. Phys. Rev. Appl. 8, 54041 (2017).

11. Kumar, S., Strachan, J. P. \& Williams, R. S. Chaotic dynamics in nanoscale $\mathrm{NbO} 2$ Mott memristors for analogue computing. Nature 548, 318 (2017).

12. Stoliar, P. et al. A leaky-integrate-and-fire neuron analog realized with a Mott insulator. Adv. Funct. Mater. 27, 1604740 (2017)

13. Lin, J., Guha, S. \& Ramanathan, S. Vanadium dioxide circuits emulate neurological disorders. Front. Neurosci. 12, 856 (2018).

14. Yi, W. et al. Biological plausibility and stochasticity in scalable VO2 active memristor neurons. Nat. Commun. 9, 4661 (2018).

15. Pickett, M. D., Medeiros-Ribeiro, G. \& Williams, R. S. A scalable neuristor built with Mott memristors. Nat. Mater. 12, 114 (2012).

16. Zhou, Y. \& Ramanathan, S. Mott memory and neuromorphic devices. Proc IEEE 103, 1289-1310 (2015).

17. del Valle, J., Salev, P., Kalcheim, Y. \& Schuller, I. K. A caloritronics-based Mott neuristor. Sci. Rep. 10, 4292 (2020).

18. Asamitsu, A., Tomioka, Y., Kuwahara, H. \& Tokura, Y. Current switching of resistive states in magnetoresistive manganites. Nature 388, 50-52 (1997).

19. Duchene, J., Terraillon, M., Pailly, P. \& Adam, G. Filamentary conduction in VO2 coplanar thin film devices. Appl. Phys. Lett. 19, 115-117 (1971).

20. Brockman, J. S. et al. Subnanosecond incubation times for electric-fieldinduced metallization of a correlated electron oxide. Nat. Nanotechnol. 9, 453 (2014).

21. Valmianski, I. et al. Origin of the current-driven breakdown in vanadium oxides: thermal versus electronic. Phys. Rev. B 98, 195144 (2018).

22. Janod, E. et al. Resistive switching in Mott insulators and correlated systems. Adv. Funct. Mater. 25, 6287-6305 (2015).

23. Stoliar, P. et al. Nonthermal and purely electronic resistive switching in a Mott memory. Phys. Rev. B 90, 45146 (2014).

24. Stoliar, P. et al. Universal electric-field-driven resistive transition in narrowgap Mott insulators. Adv. Mater. 25, 3222-3226 (2013).

25. Stefanovich, G., Pergament, A. \& Stefanovich, D. Electrical switching and Mott transition in VO2. J. Phys. Condens. Matter 12, 8837-8845 (2000).

26. Diener, P. et al. How a dc electric field drives Mott insulators out of equilibrium. Phys. Rev. Lett. 121, 16601 (2018).

27. Gopalakrishnan, G., Ruzmetov, D. \& Ramanathan, S. On the triggering mechanism for the metal-insulator transition in thin film VO2 devices: electric field versus thermal effects. J. Mater. Sci. 44, 5345-5353 (2009).

28. Simon Mun, B. et al. Role of joule heating effect and bulk-surface phases in voltage-driven metal-insulator transition in VO2 crystal. Appl. Phys. Lett. 103, 61902 (2013).

29. Kruger, B. A., Joushaghani, A. \& Poon, J. K. S. Design of electrically driven hybrid vanadium dioxide (VO2) plasmonic switches. Opt. Express 20, 23598-23609 (2012).

30. Roach, W. R. \& Balberg, I. Optical induction and detection of fast phase transition in VO2. Solid State Commun. 9, 551-555 (1971)

31. Liao, G. M. et al. Dynamically tracking the joule heating effect on the voltage induced metal-insulator transition in VO2 crystal film. AIP Adv. 6, 45014 (2016).

32. Giorgianni, F., Sakai, J. \& Lupi, S. Overcoming the thermal regime for the electric-field driven Mott transition in vanadium sesquioxide. Nat. Commun. 10, 1159 (2019).

33. Pickett, M. D. \& Stanley Williams, R. Sub-100 fJ and sub-nanosecond thermally driven threshold switching in niobium oxide crosspoint nanodevices. Nanotechnology 23, 215202 (2012).

34. Liu, M. et al. Terahertz-field-induced insulator-to-metal transition in vanadium dioxide metamaterial. Nature 487, 345-348 (2012).
35. Jager, M. F. et al. Tracking the insulator-to-metal phase transition in $\mathrm{VO} 2$ with few-femtosecond extreme UV transient absorption spectroscopy. Proc. Natl Acad. Sci. USA 114, 9558 LP-9559563 (2017).

36. Higuchi, Y., Kanki, T. \& Tanaka, H. Joule-heat-driven high-efficiency electronic-phase switching in freestanding $\mathrm{VO} 2 / \mathrm{TiO} 2$ nanowires. Appl. Phys. Express 10, 33201 (2017).

37. Guénon, $\mathrm{S}$. et al. Electrical breakdown in a $\mathrm{V} 2 \mathrm{O} 3$ device at the insulator-tometal transition. Europhys. Lett. 101, 57003 (2013).

38. del Valle, J. et al. Resistive asymmetry due to spatial confinement in first-order phase transitions. Phys. Rev. B 98, 45123 (2018).

39. Tao, Z. et al. The nature of photoinduced phase transition and metastable states in vanadium dioxide. Sci. Rep. 6, 38514 (2016).

40. Zimmers, A. et al. Role of thermal heating on the voltage induced insulator-metal transition in VO2. Phys. Rev. Lett. 110, 56601 (2013).

41. Slesazeck, S. et al. Physical model of threshold switching in $\mathrm{NbO} 2$ based memristors. RSC Adv. 5, 102318-102322 (2015).

42. Kumar, S. et al. Physical origins of current and temperature controlled negative differential resistances in NbO2. Nat. Commun. 8, 658 (2017).

43. Ielmini, D. Threshold switching mechanism by high-field energy gain in the hopping transport of chalcogenide glasses. Phys. Rev. B 78, 35308 (2008).

44. Pantelides, S. T. The electronic structure of impurities and other point defects in semiconductors. Rev. Mod. Phys. 50, 797-858 (1978).

45. Jian, J. et al. Continuous tuning of phase transition temperature in $\mathrm{VO} 2$ thin films on c-cut sapphire substrates via strain variation. ACS Appl. Mater. Interfaces 9, 5319-5327 (2017).

46. Brockman, J. et al. Increased metal-insulator transition temperatures in epitaxial thin films of $\mathrm{V} 2 \mathrm{O} 3$ prepared in reduced oxygen environments. Appl. Phys. Lett. 98, 152105 (2011).

47. Oka, T. \& Aoki, H. Ground-state decay rate for the Zener breakdown in band and Mott insulators. Phys. Rev. Lett. 95, 137601 (2005).

48. Yamakawa, H. et al. Mott transition by an impulsive dielectric breakdown. Nat. Mater. 16, 1100-1105 (2017)

49. Mayer, B. et al. Tunneling breakdown of a strongly correlated insulating state in VO2 by intense multiterahertz excitation. Phys. Rev. B 91, 235113 (2015).

50. Oka, T., Arita, R. \& Aoki, H. Breakdown of a Mott insulator: a nonadiabatic tunneling mechanism. Phys. Rev. Lett. 91, 66406 (2003).

51. Wu, B. et al. Electric-field-driven phase transition in vanadium dioxide. Phys. Rev. B 84, 241410 (2011).

52. Lee, W.-R. \& Park, K. Dielectric breakdown via emergent nonequilibrium steady states of the electric-field-driven Mott insulator. Phys. Rev. B 89, 205126 (2014)

53. Sugimoto, N., Onoda, S. \& Nagaosa, N. Field-induced metal-insulator transition and switching phenomenon in correlated insulators. Phys. Rev. B 78, 155104 (2008)

54. Yang, Z., Hart, S., Ko, C., Yacoby, A. \& Ramanathan, S. Studies on electric triggering of the metal-insulator transition in $\mathrm{VO} 2$ thin films between $77 \mathrm{~K}$ and 300 K. J. Appl. Phys. 110, 33725 (2011).

55. Frenkel, J. On pre-breakdown phenomena in insulators and electronic semiconductors. Phys. Rev. 54, 647-648 (1938)

56. Qazilbash, M. M. et al. Electrodynamics of the vanadium oxides VO2 and V2O3. Phys. Rev. B 77, 115121 (2008)

57. Ieda, M., Sawa, G. \& Kato, S. A consideration of Poole-Frenkel effect on electric conduction in insulators. J. Appl. Phys. 42, 3737-3740 (1971).

58. Schroeder, H. Poole-Frenkel-effect as dominating current mechanism in thin oxide films-an illusion?! J. Appl. Phys. 117, 215103 (2015).

59. Ganichev, S. D. et al. Distinction between the Poole-Frenkel and tunneling models of electric-field-stimulated carrier emission from deep levels in semiconductors. Phys. Rev. B 61, 10361-10365 (2000).

60. Kim, H.-T. et al. Electrical oscillations induced by the metal-insulator transition in VO2. J. Appl. Phys. 107, 23702 (2010).

61. Georges, A., Kotliar, G., Krauth, W. \& Rozenberg, M. J. Dynamical mean-field theory of strongly correlated fermion systems and the limit of infinite dimensions. Rev. Mod. Phys. 68, 13-125 (1996).

62. Taillefer, L. Scattering and pairing in cuprate superconductors. Annu. Rev. Condens. Matter Phys. 1, 51-70 (2010).

63. Zhang, Z. et al. Evolution of metallicity in vanadium dioxide by creation of oxygen vacancies. Phys. Rev. Appl. 7, 34008 (2017).

64. Shivashankar, S. A. \& Honig, J. M. Metal-antiferromagnetic-insulator transition in V2O3 alloys. Phys. Rev. B 28, 5695-5701 (1983).

65. Yang, J. J. et al. High switching endurance in TaOx memristive devices. Appl. Phys. Lett. 97, 232102 (2010).

66. del Valle, J. et al. Subthreshold firing in Mott nanodevices. Nature 569, 388 (2019).

67. Trastoy, J., Kalcheim, Y., del Valle, J., Valmianski, I. \& Schuller, I. K. Enhanced metal-insulator transition in $\mathrm{V} 2 \mathrm{O} 3$ by thermal quenching after growth. J. Mater. Sci. 53, 9131-9137 (2018). 


\section{Acknowledgements}

This research was supported through an Energy Frontier Research Center funded by the U.S. Department of Energy, Office of Science, Basic Energy Sciences under Award no. DE-SC0019273. Part of the fabrication process was done at the San Diego Nanotechnology Infrastructure (SDNI) of UCSD, a member of the National Nanotechnology Coordinated Infrastructure (NNCI), which is supported by the US National Science Foundation under grant ECCS-1542148. A.C. gratefully acknowledges support from CONICET and UBACyT.

\section{Author contributions}

Y.K. and I.K.S. conceived and designed the experiment. Y.K., P.S., and J.d.V. fabricated the samples. Y.K. performed the transport measurements, focused ion beam irradiation, and Joule heating simulations. A.C. and M.J.R. performed DMFT calculations. Y.K. wrote the manuscript. All authors participated in discussion of the results and in writing multiple iterations of the manuscript.

\section{Competing interests}

The authors declare no competing interests.

\section{Additional information}

Supplementary information is available for this paper at https://doi.org/10.1038/s41467020-16752-1.
Correspondence and requests for materials should be addressed to Y.K.

Peer review information Nature Communications thanks Marek Skowronski and the other, anonymous, reviewer(s) for their contribution to the peer review of this work.

Reprints and permission information is available at http://www.nature.com/reprints

Publisher's note Springer Nature remains neutral with regard to jurisdictional claims in published maps and institutional affiliations.

(c) (i) Open Access This article is licensed under a Creative Commons Attribution 4.0 International License, which permits use, sharing, adaptation, distribution and reproduction in any medium or format, as long as you give appropriate credit to the original author(s) and the source, provide a link to the Creative Commons license, and indicate if changes were made. The images or other third party material in this article are included in the article's Creative Commons license, unless indicated otherwise in a credit line to the material. If material is not included in the article's Creative Commons license and your intended use is not permitted by statutory regulation or exceeds the permitted use, you will need to obtain permission directly from the copyright holder. To view a copy of this license, visit http://creativecommons.org/licenses/by/4.0/.

(c) The Author(s) 2020 